\title{
Modelo de referência para estruturar o Seis Sigma nas organizações
}

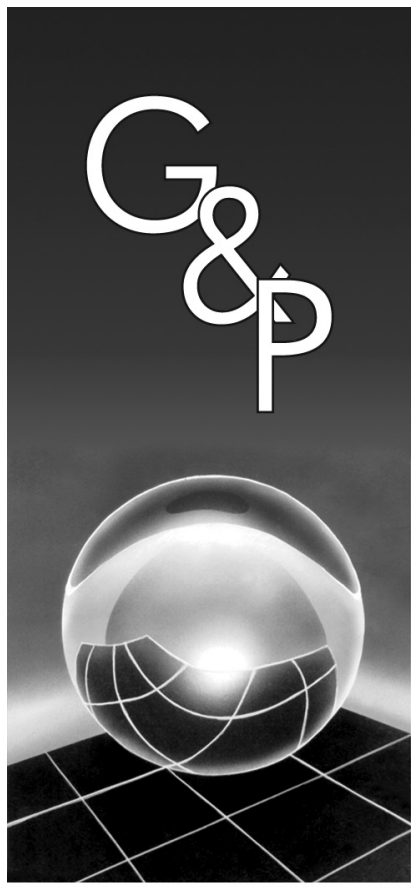

Adriana Barbosa Santos

Manoel Fernando Martins

\section{Resumo}

Este artigo apresenta o modelo de referência para estruturar o Seis Sigma, o qual é resultante da incorporação de teorias que contribuem para aumentar o potencial estratégico do Seis Sigma no sentido de incrementar o desempenho organizacional. Em sua proposta, o modelo de referência engloba um direcionamento sobre certos requisitos primordiais para o sucesso do programa Seis Sigma. A base teórica de sustentação do modelo de referência foi construída a partir de estudos sobre a influência dos seguintes fatores: orientação estratégica e alinhamento estratégico; medição e gerenciamento do desempenho organizacional; uso de estatística (pensamento estatístico); capacitação/especialização de pessoas; implementação e gerenciamento de projetos; e uso de tecnologia de informação. Complementando a proposição do modelo, o artigo traz evidências empíricas acerca da contribuição dos fatores identificados na formulação do modelo de referência, expondo resultados decorrentes de estudos de caso realizados em quatro subsidiárias brasileiras de multinacionais de grande porte. A análise dos dados forneceu evidências positivas de que os fatores mencionados influenciam de forma efetiva o sucesso e a consolidação do Seis Sigma nas empresas estudadas.

Palavras-chave: Seis Sigma. Qualidade. Alinhamento estratégico. Medição de desempenho. DMAIC. Pensamento estatístico.

\section{Introdução}

A descrição de vários casos bem sucedidos sobre os resultados dosinvestimentos no Seis Sigma(CORONADO; ANTONY, 2002) despertou o interesse sobre o assunto nas organizações dos diversos setores industriais pelo fato de possibilitar não apenas a melhoria da qualidade de produtos, serviços e processos, como também por possibilitar um incremento significativo no desempenho organizacional, na mudança de cultura e no aumento do capital humano.

Frente à necessidade crescente de buscar a diminuição dos custos de produção, a eliminação de desperdícios e a redução da variabilidade nos processos críticos para o negócio, o pensamento estatístico e os métodos estatísticos passaram a ser valorizados como meios vitais para atingir os objetivos estratégicos de algumas organizações, tendo o alinhamento estratégico como catalisador.

Ao longo da última década, o Seis Sigma veio se consolidando como uma abordagem abrangente que está alinhada à implementação de estratégias que promovem a melhoria do desempenho do negócio, aumentando o potencial competitivo e impulsionando as ações estratégicas e gerenciais que: (a) priorizem a melhoria contínua do nível de qualidade de produtos e/ou serviços; (b) incrementem a capacidade de inovação, mesmo diante da dificuldade de estabelecer vantagens competitivas; e (c) reduzam custos e desperdícios. Em face de suas implicações, essas ações organizacionais estão ganhando cada vez mais destaque e atenção, não apenas junto à comunidade acadêmica, mas também no meio empresarial (SANTOS, 2006). Estes aspectos sugerem que a implementação do programa Seis Sigma seja tratada como um tema amplo, ajustado às ações estratégicas mencionadas acima, e com abrangência que vá além da aplicação de uma metodologia sistematizada para implementação de projetos.

Neste contexto, este artigo tem como objetivo principal apresentar o modelo de referência, o qual resulta da incorporação de teorias que contribuem para aumentar o potencial estratégico do Seis Sigma no sentido de incrementar o desempenho organizacional.

A consistência teórica do modelo de referência decorre de uma visão conceitual que combina tanto a abordagem estatística como a abordagem estratégica do Seis Sigma, 
e também da análise da influência de fatores como: orientação estratégica e alinhamento estratégico; medição e gerenciamento do desempenho organizacional; uso de estatística (pensamento estatístico); capacitação/especialização das pessoas; implementação e gerenciamento de projetos; e uso de tecnologia de informação. Devido ao impacto que cada um destes fatores pode exercer para aumentar a eficiência e a eficácia dos processos produtivos, eles foram reunidos na construção do modelo de referência.

Complementando a descrição da importância dos fatores citados acima, em âmbito teórico são apresentados resultados decorrentes de uma pesquisa empírica que buscou compreender como estes fatores influenciam o sucesso do Seis Sigma, bem como as ações gerenciais e estratégicas. As evidências empíricas foram obtidas com base no método do estudo de caso (múltiplos casos), os quais foram conduzidos em quatro subsidiárias brasileiras de multinacionais de grande porte. A análise cruzada dos dados fornecidos por pessoas diretamente envolvidas com o Seis Sigma nas empresas revelou aspectos que valorizam o modelo aqui proposto.

\section{Seis Sigma: abordagens, definições e fatores críticos de sucesso}

Ao examinar a literatura sobre Seis Sigma, é possível perceber que o assunto sofreu uma evolução conceitual, principalmente no que toca à visão e à identificação de quais seriam os fatores chave que influenciam o sucesso do Seis Sigma na maioria das organizações.

Com relação à visão geral do Seis Sigma, duas abordagens podem ser identificadas mais claramente na literatura: a abordagem estatística e a abordagem estratégica. Na primeira, o foco está na quantificação da variação, que é feita em quantidades de desvios padrão $(\sigma)$ associados a uma variável aleatória de interesse no estudo de um processo crítico. Esta visão está fortemente relacionada aos conceitos de controle estatístico de processos (CEP), por isso é comum encontrar na literatura muitas referências ao Seis Sigma descrevendo a relação dos índices de capacidade de processo com o padrão $6 \sigma$, bem como esclarecimentos sobre a origem do valor 3,4 PPM (partes por milhão), como sendo um valor descritivo da quase perfeição e do padrão de qualidade $6 \sigma$.

Até o final dos anos 90, a maior parte das publicações sobre o tema discutiu com maior ênfase os aspectos metodológicos inseridos na implementação de projetos. Com o passar do tempo, os reflexos produzidos no desempenho do negócio ganharam mais destaque, fazendo com que os pesquisadores passassem a incluir aspectos da abordagem estratégica como foco da definição do Seis Sigma.
A Figura 1 destaca algumas definições de Seis Sigma selecionadas da literatura com o intuito de ilustrar essa evolução conceitual do Seis Sigma. De fato, a abordagem estratégica do Seis Sigma passou a ser mais empregada na virada do século, quando se nota que Seis Sigma começou a ser compreendido como "estratégia", complementando seu significado metodológico.

A definição produzida por Santos (2006), além de explicitar elementos da gestão da qualidade não apontados por outros autores, salienta ainda mais o enfoque estratégico dado ao Seis Sigma na atualidade.

A Tabela 1 traz resultados quantitativos obtidos em decorrência da revisão da literatura. Note que a tabela apresenta o número de publicações que destacam certos fatores críticos do sucesso do Seis Sigma estratificados para dois períodos com amplitudes diferentes. Os valores apresentados correspondem à quantidade de publicações cujo foco principal pôde ser classificado em pelo menos uma das categorias de enfoque apontadas. Foram analisadas aproximadamente 50 publicações, incluindo artigos e livros; o que pode ser considerada uma revisão parcial, porém representativa das tendências sobre um assunto que ainda não foi explorado exaustivamente pelos pesquisadores.

Pela Figura 1 e Tabela 1 pode-se perceber que há uma tendência das publicações em reforçar as implicações gerenciais e estratégicas que permeiam a implementação do Seis Sigma. O enfoque estatístico, tão enfatizado nas publicações do início da década de 90, continua sendo priorizado, no entanto, de forma mais restrita à aplicação da metodologia sistematizada pelo ciclo DMAIC (definir; medir; analisar; incrementar; e controlar) e pelo DFSS (design for Six Sigma). Estes tópicos integram boa parte das discussões encontradas na literatura acerca da implementação dos projetos Seis Sigma e da especialização das pessoas. O DMAIC é mais caracterizado pelo seu potencial de solução de problemas por assegurar a redução na taxa de defeitos e falhas nos produtos, serviços e processos. Já o DFSS pelo seu enfoque mais preventivo é mais direcionado para a inovação e otimização e tem sido uma solução potente na minimização de ocorrências indesejáveis e inconveniências associadas ao lançamento e desenvolvimento de novos produtos, bem como no reprojeto de novos processos. A implementação do Seis Sigma inclui o uso dessas duas abordagens metodológicas.

O pensamento estatístico aparece como um tópico mais recente à medida que envolve uma visão mais holística de aplicação dos fundamentos estatísticos. A relação com a implementação da estratégia do negócio e com o uso dos indicadores de desempenho também é discutida na literatura nas publicações analisadas, uma vez que o Seis Sigma foi sendo compreendido pelos pesquisadores como um programa amplo que pode estar interligado aos 


Abordagem estatística
"Iniciativa chave que dá suporte à companhia no seu plano de
satisfação total do cliente". (MITCHELL, 1992)

"É um modo de medir a probabilidade de produzir um produto ou criar um serviço com zero defeito." (TADIKAMALLA, 1994)

"É uma maneira de medir a probabilidade de a companhia poder fabricar ou produzir qualquer dada unidade de um produto ou serviço com zero defeito. É a categoria que significa "best in class", com somente 3,4 DPMO". (BEHARA et al., 1995)

\section{Abordagem estratégica}

"É um processo de negócio que permite à companhia melhorar drasticamente seus limites inferiores, projetando e monitorando diariamente as atividades do negócio de uma maneira que minimizem o desperdício e os recursos enquanto aumentam a satisfação do cliente". (HARRY; SCHROEDER, 2000)

\section{"É uma abordagem de melhoria de negócio que busca achar} e eliminar causas de falhas e defeitos no processo de negócio, focando sobre as saídas que são de importância crítica para os clientes. É uma abordagem estratégica que trabalha através de todos os processos, produtos, funções da companhia e indústrias". (SNEE, 2000)

"Estratégia que abastece as companhias com uma série de intervenções e ferramentas estatísticas que podem levar a ganhos substanciais em lucratividade e qualidade, tanto para produtos como serviços". (HARRY, 1998)

"É uma abordagem quantitativa disciplinada para melhoria de métricas definidas em processos de manufatura, serviço ou financeiro". (HOERL, 1998)

"É uma abordagem de alto desempenho, direcionada para dados para analisar as causas raízes dos problemas do negócio a fim de resolvê-los. Ela amarra os resultados de um negócio aos requisitos de mercado". (BLAKESLEE, 1999)

"Um sistema abrangente e flexível para alcançar, sustentar e maximizar o sucesso empresarial. É singularmente impulsionado por uma estreita compreensão das necessidades dos clientes, pelo uso disciplinado de fatos, dados e análise estatística e a atenção diligente à gestão, melhoria e reinvenção dos processos de negócios". (PANDE et al., 2001)

"Uma estratégia para melhoria de negócios usada para melhorar a lucratividade do negócio, eliminar refugo, reduzir custo da não qualidade e melhorar a eficiência e eficácia de todas as operações, assim como encontrar, ou mesmo exceder as expectativas e necessidades dos clientes". (CORONADO; ANTONY, 2002)

"É um nível otimizado de performance que se aproxima do zero defeito em um processo de confecção de um produto, serviço ou transação. Ele indica a obtenção e a manutenção de uma performance de alto nível. O Seis Sigma não é uma metodologia. É um fim, não um meio". (PEREZ-WILSON, 1999)

"Para nós, Seis Sigma é mais do que uma metodologia e um conjunto de ferramentas. É também um modo de pensar que possibilita-nos mudar o modo de trabalho para torná-lo mais dirigido aos dados. Seis sigma afasta-nos da decisão baseada na intuição". (MOTWANI et al., 2004)

"Seis Sigma é baseado nas velhas idéias de engenharia da qualidade destinadas a entender e eliminar as causas de variação e projetar a manufatura". (DALE et al., 2000)

"Hoje, Seis Sigma é uma estratégia abrangente de longo prazo para tomada de decisão mais do que um programa estritamente focado na gestão da qualidade" (ARNHEITER ; MALEIYEFF, 2005)

"Seis Sigma é uma abordagem que impulsiona a melhoria do desempenho do negócio e a valorização da satisfação dos clientes, por meio do enfoque estratégico de gerenciamento; da aplicação do pensamento estatístico em todos os níveis de atividades; da medição de desempenho; da utilização de uma metodologia sistematizada que integre técnicas e métodos científicos para se avaliar e otimizar processos; e da aprendizagem decorrente da capacitação e comprometimento das pessoas". (SANTOS, 2006)

Figura 1. Definições de Seis Sigma.

Tabela 1. Fatores críticos do sucesso do Seis Sigma destacados na literatura. (*Fonte: textos digitais completos consultados em: www.emeraldinsight.com; www.scielo.org; www.springerlink.com; www.asq.org; www.googlescholar.com; anais do SIMPOI 2005; anais do ENEGEP de 2002 a 2005; e livros impressos.)

\section{Enfoque da publicação}

Número de publicações por período*

Impacto no cliente; orientação para o mercado; e alinhamento estratégico

Abordagem estratégica e gerencial

Medição de desempenho

Comprometimento da liderança

Pensamento estatístico; metodologia Seis Sigma (DMAIC/DFSS); e projetos Seis Sigma De 1992 a 2000 De 2001 a 2006

Capacitação e aprendizagem

$\begin{array}{cc}5 & 6 \\ 7 & 10 \\ 4 & 3 \\ 3 & 3 \\ 11 & 15 \\ 6 & 7\end{array}$

assuntos estratégicos e ao impacto sobre o desempenho do negócio. O meio adotado para capacitação das pessoas ainda é um tópico abordado com limitações, sem a introdução de novas teorias para avaliar os reflexos positivos ou negativos gerados pelos cursos de treinamentos dos membros da equipe de especialistas (master black belts; black belts; e green belts).

\section{0 modelo de referência}

Nas organizações que investem no Seis Sigma, a redução da variação, a medição e a coleta de dados podem ser interpretadas como um princípio básico para o sucesso do Seis Sigma. Todavia, o sucesso do programa Seis Sigma depende também de uma combinação harmo- 
niosa de certos fatores considerados essenciais na sua implementação.

A Figura 2 introduz um esquema de representação do modelo de referência. Este modelo fornece um direcionamento sobre requisitos, que podem ser primordiais para que uma organização possa estruturar ou mesmo reestruturar a implementação do programa Seis Sigma. A estrutura representada nesta figura traz em destaque a orientação e o alinhamento estratégico; a medição e o gerenciamento do desempenho organizacional; o uso de estatística (pensamento estatístico); a capacitação e a especialização das pessoas; e a implementação e o gerenciamento de projetos, como fatores essenciais para estruturar ou reestruturar o processo de implantação do programa Seis Sigma. Na verdade, a Figura 2 ilustra a estrutura do modelo, de modo que o Seis Sigma seja considerado um programa amplo, que expressa o modo e o plano traçado pela organização para atingir seus objetivos estratégicos e operacionais de melhoria da qualidade e de aumento de potencial competitivo, por meio da maior satisfação dos clientes.
Os fatores identificados no modelo de referência são detalhados nos subtópicos apresentados a seguir.

\subsection{O Programa Seis Sigma}

Um dos aspectos fundamentais para compreender a Figura 2 é perceber que o Seis Sigma deve ser entendido como um programa estratégico e também operacional, que promove a integração entre os objetivos estratégicos e os objetivos operacionais. Como programa operacional, o Seis Sigma impulsiona a melhoria contínua afetando mais diretamente as áreas em que não haja trade-offs. Já como programa estratégico, está direcionado para o ambiente, buscando um incremento na posição competitiva, evidenciando os pontos de trade-offs e impondo um feedback estratégico frente às metas financeiras incorporadas ao escopo dos projetos.

Por estar embasado no gerenciamento por processos e por diretrizes, e pelo seu papel de integrador, o Seis Sigma reafirma a necessidade de interligação entre os objetivos estratégicos e os objetivos operacionais, fazendo com que uma comunicação estratégica efetiva seja necessária à medida que os projetos Seis Sigma são definidos.

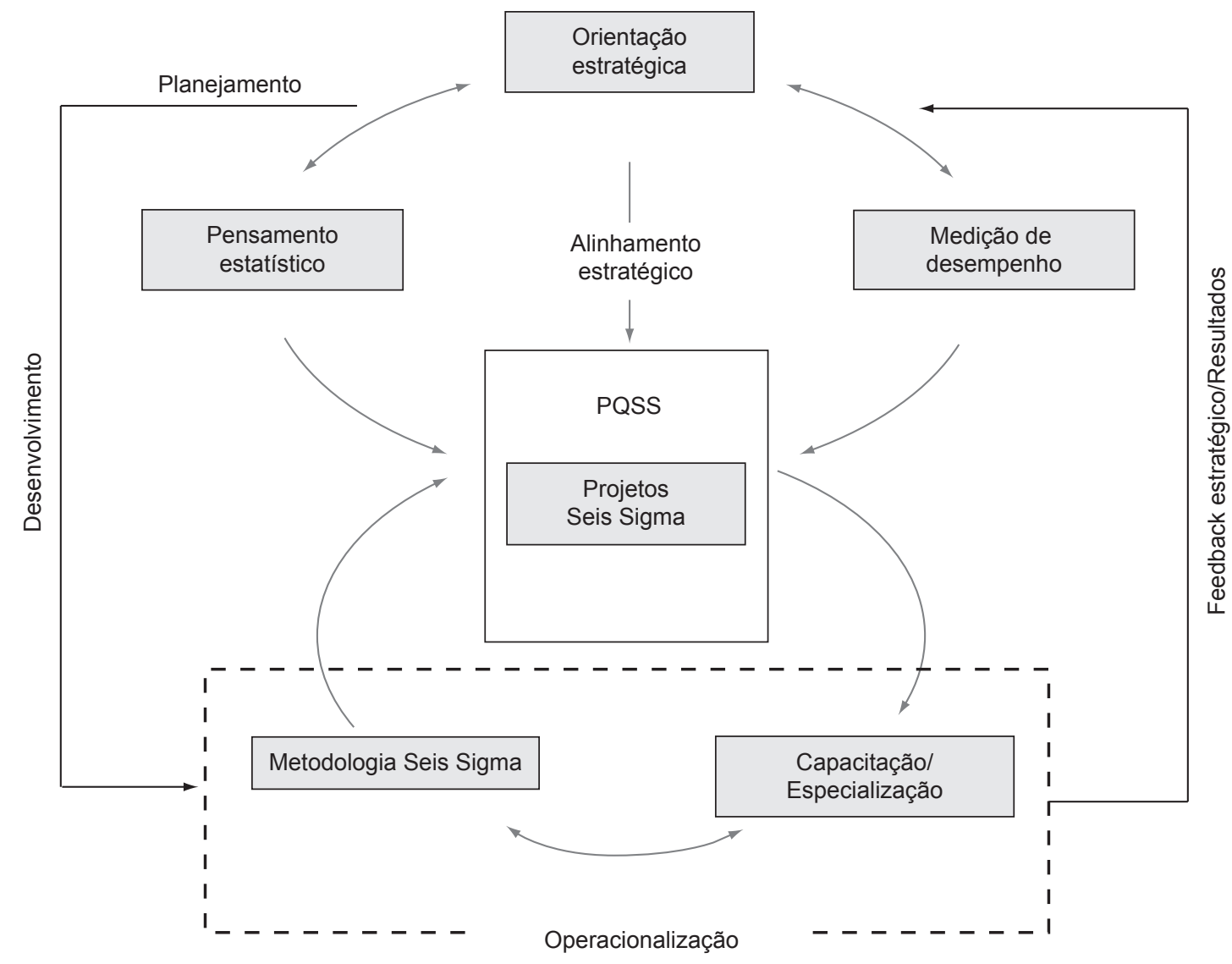

Figura 2. Esquema da estrutura do Modelo de Referência. 
Em face destes aspectos, o fato dos responsáveis pela implementação dos projetos e pela disseminação da cultura Seis Sigma conhecerem e compreenderem quais são os objetivos estratégicos traçados pela alta administração contribui para que eles identifiquem quais ações são necessárias para alcançá-los e, a partir deste ponto, estabeleçam objetivos e metas operacionais alinhados estrategicamente.

De fato, a implementação dos projetos Seis Sigma constitui uma aplicação imediata dessa integração, visto que eles representam oportunidades de melhoria significantes para que a estratégia do negócio seja implementada. A combinação da integração com alinhamento estratégico e o desempenho configura um tripé de relacionamento que tanto afeta como é afetado diretamente pela implementação do programa Seis Sigma.

\subsection{Projetos Seis Sigma}

Um projeto Seis Sigma visa reduzir a variabilidade e aumentar a capacidade dos processos. Sua implementação está vinculada ao uso de métodos estatísticos que buscam facilitar a interpretação de relações de causa e efeito que afetam diretamente processos críticos para o negócio.

$\mathrm{Na}$ estrutura do modelo de referência, os projetos Seis Sigma são catalisadores de um sistema, permitem o feedback operacional e, sobretudo, o feedback estratégico que auxilia na revisão e/ou redirecionamento de metas de médio e longo prazo. Por isso, seus resultados influenciam significativamente a tomada de decisão, tanto em nível estratégico, como em nível tático e operacional. Pela ligação que podem estabelecer com os indicadores de desempenho eles têm um papel de direcionadores de desempenho (SANTOS; MARTINS, 2005). Seus resultados implicam em ganhos significativos e tangíveis sobre algumas dimensões/categorias de desempenho predefinidas na composição do sistema de medição de desempenho organizacional.

\subsection{Orientação estratégica e alinhamento estratégico}

A orientação estratégica e o alinhamento estratégico são fatores que têm papel primordial no direcionamento e na priorização do foco da implementação dos projetos Seis Sigma e do programa Seis Sigma como um todo.

O alinhamento estratégico é um fator chave para a consistência de propósitos e ações diante das iniciativas de melhoria (McADAM; BAILIE, 2002) e, para as organizações que investem no Seis Sigma, o programa se constitui num catalisador desse alinhamento, pelo fato de ser um programa estratégico que promove a melhoria de todo o negócio, a partir do alcance dos objetivos estratégicos. Com a implementação do programa estando vinculada à necessidade de alinhamento estratégico, o
Seis Sigma influencia a formulação e a implementação da estratégia competitiva, à medida que fornece indicativos sobre as diretrizes estratégicas seguidas na formulação dos projetos.

Valendo-se de uma abordagem predominantemente quantitativa, o Seis Sigma usa medição e análise de dados como o principal meio de captura de informação para qualquer tomada de decisão, desde a definição dos projetos Seis Sigma até o direcionamento dos resultados de desempenho propiciados pela conclusão destes projetos (REVERE; BLACK, 2003).

No âmbito do modelo de referência, os projetos Seis Sigma são originados nas prioridades estabelecidas pela alta administração, portanto, fornecem indicativos de que a implementação do programa Seis Sigma é direcionada pelos objetivos e metas estratégicas. Além disso, a definição destes projetos obedece a um direcionamento pré-estabelecido na estratégia de desdobramento do Seis Sigma adotada pela organização. Isto fica mais evidente à medida que o escopo dos projetos é elaborado e detalhado.

Outro ponto relevante implícito na concepção do modelo é que ele absorve aspectos relativos ao impacto da orientação estratégica sob a gestão da qualidade total (DANSKY; BRANNON, 1996). A conscientização das pessoas sobre as metas de longo prazo, sobre como as ações individuais contribuem para a realização dos objetivos estratégicos, de haver uma direção comum e, sobretudo, de se dispor de uma gramática de comunicação que facilite este alinhamento. Deste modo, dispor de mecanismos para uma comunicação efetiva traz um incremento para que as pessoas possam intercambiar informações e compreendê-las. Compartilhá-las é uma forma de impulsionar o aumento do comprometimento das pessoas para obter alinhamento.

\subsection{Medição de desempenho}

A medição de desempenho é um tópico inserido nas diversas iniciativas de melhoria que foram adotadas pelas organizações nas últimas décadas, mais especificamente com respeito aos parâmetros relativos à qualidade, custo e tempo de ciclo. Com o Seis Sigma esse assunto se tornou ainda mais essencial para as organizações medirem seus resultados de desempenho. Pelo fato de ser uma abordagem dirigida para a medição e a tomada de decisão baseada em dados e fatos, o Seis Sigma vem contribuindo para o aprimoramento da medição de desempenho, tornando-a um pré-requisito para a melhoria contínua dos processos e para a implementação da estratégia competitiva.

De fato, a essência da melhoria contínua está no conhecimento que a organização tem sobre o que é necessário melhorar, bem como sobre os indicadores financeiros e não financeiros que refletem o desempenho organizacional (NEELY, 1998; 1999). Para isso, 
é preciso identificar "onde" e "por que" o desempenho de momento não é satisfatório, para que então os indicadores de desempenho dêem o suporte necessário no que diz respeito ao direcionamento das ações de melhoria.

McAdam e Bailie (2002) fornecem uma amostra das vantagens do Seis Sigma em relação aos outros programas de qualidade já há mais tempo difundidos na literatura, ao conseguirem resultados práticos relevantes sobre os efeitos positivos do Seis Sigma, quando comparado a outras iniciativas de melhoria, como: gestão da qualidade total; programa de gestão do desempenho; manutenção produtiva total; entre outras. Por meio de um estudo de caso prolongado, os autores mostram que o Seis Sigma e o programa de gestão do desempenho são as iniciativas que mais contribuem para o alinhamento da medição de desempenho com a estratégia.

Por sua abordagem quantitativa, o Seis Sigma tende a ser um programa rico em indicadores de desempenho, que na sua maioria são direcionadores de desempenho adequados para melhorar o alinhamento com a estratégia (EVANS, 2004). O uso de indicadores de desempenho é imprescindível para compreender que o gerenciamento das ações envolvidas no programa Seis Sigma é parte do gerenciamento do desempenho, e que os indicadores podem ser usados não apenas para controlar a implementação da estratégia de negócio, mas também podem ser usados para direcionar os projetos Seis Sigma.

A integração das ações planejadas estrategicamente requer o desenvolvimento de mecanismos para integrar e sustentar o foco da organização na estratégia do negócio, monitorar e gerenciar as expectativas dos clientes e gerenciar o desempenho para atender a essas expectativas (CROSS; LYNCH, 1990). Neste sentido, o uso de um sistema de medição de desempenho (SMD) multidimensional, baseado em indicadores financeiros e não financeiros, é fundamental para avaliar as dimensões de desempenho mais afetadas pelos processos críticos que deverão ser analisados com mais detalhes no Seis Sigma.

A relação da medição do desempenho com os projetos Seis Sigma, representada no modelo de referência, fica mais evidente à medida que se entende que os resultados dos projetos contribuem para o gerenciamento do desempenho, pois permitem a identificação de direcionadores de desempenho importantes para a revisão e/ou redefinição dos objetivos estratégicos, conforme já discutiram Santos e Martins (2003).

\subsection{Pensamento estatístico}

O pensamento estatístico consiste num elemento básico e fundamental para alicerçar as ações no contexto do Seis Sigma em todos os níveis de atividades. Conforme já apontado por Santos e Martins (2004), no contexto do Seis Sigma a aplicação do pensamento estatístico, a priorização do foco em processos, o entendimento da variação e o uso de dados para subsidiar ações devem ser entendidos como elementos determinantes do sucesso das atividades diárias de gerenciamento de processos e da implementação dos projetos.

O pensamento estatístico incrementa o uso das ferramentas estatísticas e gerenciais que visam à melhoria da qualidade dos produtos e processos; por ser uma abordagem que contribui para tornar a tomada de decisão um reflexo de ações gerenciais enriquecidas por subsídios mais consistentes; e pelo aumento do valor da tomada de decisão (DRANSFIELD et al., 1999; GOH, 2002; GOH; XIE, 2004).

Medir e coletar dados sobre o processo desde que sejam necessários e suficientes para suportar decisões; analisar as fontes de variação que afetam processos, pessoas, materiais, métodos, máquinas e ambiente; procurar a simplicidade sem ignorar a existência de variabilidade; desenvolver e conscientizar as pessoas; buscar a melhoria contínua e a perfeição; e aumentar a credibilidade das informações foram princípios introduzidos por Santos (2006), que fortalecem a estrutura do modelo de referência. Seu potencial está em facilitar a compreensão e uso da estatística e, naturalmente, na disseminação da metodologia sistematizada prescrita para implementar os projetos Seis Sigma. Além disso, o modelo de referência pode ser usado para criar uma cultura que deve ser difundida para todos os funcionários da organização e que esteja engajada na implementação do programa Seis Sigma (ANTONY, 2004).

\subsection{Metodologia sistematizada para solução de problemas}

A aplicação da metodologia sistematizada Seis Sigma reafirma o valor do método científico, em especial do método estatístico como forma eficiente de eliminar a causa raiz dos problemas para garantir a obtenção de resultados concretos. O enfoque metodológico do Seis Sigma é o tópico mais discutido na literatura, conforme pôde ser visualizado na Tabela 1, pois trata mais especificamente da utilização de métodos estatísticos empregados numa seqüência metodológica sistematizada de acordo com os ciclos de melhoria DMAIC (define; measure; analyse; improve; e control) e DMADV (define; measure; analyse; develop; e verify). A metodologia Seis Sigma, como é conhecida, não requer apenas uma base sólida de conhecimentos estatísticos. Outros métodos científicos quantitativos para solução de problemas, controle e otimização de processos são muito proveitosos (HAHN et al., 2000).

A eficiência da metodologia sistematizada Seis Sigma já foi comprovada por várias organizações de classe mundial como uma iniciativa de melhoria contínua da qualidade dos processos, produtos e serviços (KWAK; ANBARI, 2006). A aplicação eficiente da metodologia 
requer sinergia entre a organização e as pessoas, a fim de possibilitar que a equipe envolvida esteja pelo menos minimamente preparada com relação à fundamentação conceitual sobre estatística, extração de conhecimento a partir de dados quantitativos e qualitativos, conhecimento sobre a dinâmica dos processos, entre outros. Somente assim, a melhoria de desempenho almejada será realmente atingida.

Embora proposto como um componente mais diretamente ligado às atividades de nível operacional dentro da estrutura do modelo de referência, a aplicação da metodologia Seis Sigma está vinculada e influenciada pelos efeitos benéficos da assimilação dos princípios do pensamento estatístico. Ambos influenciam a implementação dos projetos, sendo que um tem um impacto maior na concepção e definição (pensamento estatístico) e outro na execução (metodologia Seis Sigma).

\subsection{Capacitação/especialização das pessoas}

Ao se incorporar a capacitação/especialização das pessoas no modelo de referência, a retenção do conhecimento gerado pelo aprimoramento intelectual e técnico está sendo considerada como um dos fatores determinantes de sucesso do Seis Sigma. Além disso, uma vez que a capacidade criativa e a motivação das pessoas são ativos valiosos para qualquer organização, podendo em alguns aspectos serem considerados parte integral da tecnologia da empresa, o investimento na formação e capacitação das pessoas se reverte num incremento ao capital intelectual (VALLE, 1996; EDVINSSON, 1998; SULLIVAN, 1999).

Note-se que um dos diferenciais do Seis Sigma, em termos de investimentos em recursos humanos, foi a criação de uma equipe de pessoas mais aptas a desenvolverem e promoverem o aumento do desempenho organizacional, a partir dos resultados da implementação de projetos direcionados estrategicamente. A definição dessa equipe composta por pessoas com papéis distintos e níveis de subordinação diferenciados consiste numa forma de reunir colaboradores para que a organização consiga incrementar significativamente o desempenho de seus processos e para que aumente o padrão de qualidade de seus produtos de modo perceptível pelo cliente e desenvolva seu potencial de aprendizagem.

Compor uma equipe que responda pela infra-estrutura criada para implementação do programa nem sempre é tarefa fácil, haja vista que não basta apenas possuir habilidades técnicas ou de solução de problemas (GEORGE, 2004). Características como liderança inata são determinantes em algumas posições dentro da estrutura do Seis Sigma, como nos casos dos master black belts, champion e black belts.

A necessidade de sintonia da equipe com os fatores competitivos não é restrita às atribuições dos dirigentes
(HANH et al., 2000), mas são eles os disseminadores da visão e da cultura da qualidade, assim como são pessoas das quais se espera participação ativa nas atividades e contribuição na melhor de suas capacidades. Além disso, os especialistas respondem pelos resultados decorrentes do sucesso ou não dos projetos; e podem contribuir como consultores internos em diversas situações.

Neste sentido, o Seis Sigma trouxe um novo cenário para os requisitos de habilitação dos profissionais que atuam mais diretamente com objetivos de melhoria da qualidade (HOERL, 2001), face ao fato que precisam estar sintonizados com as muitas funções da empresa para estabelecerem parâmetros financeiros e operacionais aos projetos sob sua responsabilidade. Mais ainda, eles devem estar atentos e preparados para trabalharem com uma vasta base de dados e informações e, para isso, a ampliação do conhecimento deve ser uma prática constante.

A capacidade dos líderes da empresa de perceberem a qualidade como um impulsionador para o traçado de objetivos estratégicos como um requisito essencial para o sucesso da implementação do programa Seis Sigma é outro ponto de destaque. Uma organização se torna funcional por meio do Seis Sigma se ela adota uma abordagem que seja voltada, simultaneamente, para clientes, processos e funcionários (ECKES, 2001).

$\mathrm{Na}$ estrutura do modelo de referência, a retenção do conhecimento proveniente da capacitação/especialização da equipe consiste num dos pilares de sustentação do Seis Sigma, pois o sucesso do programa é interdependente do comprometimento inflexível da alta gerência (HAHN et al., 2000; BLAKESLEE, 1999).

\section{Aspectos metodológicos da pesquisa empírica}

O suporte empírico do trabalho foi obtido por meio de uma abordagem de pesquisa qualitativa (CRESWELL, 1994), em que se empregou o método do estudo de caso (múltiplos casos) como procedimento de coleta de dados (BRYMAN, 1989; YIN, 2005). Os estudos de caso foram conduzidos com o intuito de se obter resposta para a seguinte questão de pesquisa: como a implementação do programa Seis Sigma é influenciada pela orientação e alinhamento estratégico; medição de desempenho; uso de estatística; formação e capacitação das pessoas; tecnologia da informação (TI); entre outros investimentos que afetam as ações gerenciais e estratégicas?

Realizada no ano de 2005, a coleta dos dados foi feita por meio de entrevistas semi-estruturadas, nas quais especialistas black belts e green belts foram as fontes de evidências principais. Informações decorrentes de observações diretas realizadas por um dos autores nas visitas às empresas também serviram de subsídio complementar. As entrevistas foram baseadas em um roteiro de pesquisa 
contendo um total de 40 perguntas, sendo que a maioria delas foi formulada visando uma melhor compreensão sobre os fatores que influenciam a implementação do programa. Algumas perguntas foram adicionadas para garantir a abrangência descritiva desejada pelos autores. Dentre os tópicos abordados constam: (i) descrição e caracterização da empresa; (ii) aspectos relativos à filosofia, à definição de Seis Sigma e ao motivo dos investimentos no Seis Sigma; (iii) características gerais do programa Seis Sigma: suas abordagens, estrutura de implementação e gerenciamento; (iv) a formação dos especialistas, o comprometimento e o gerenciamento dos participantes e responsáveis pelas atividades do Seis Sigma; (v) gerenciamento dos projetos Seis Sigma, o uso da medição de desempenho e forma de controle de resultado; e (vi) os fatores de sucesso e os fatores de risco associados à implementação do programa Seis Sigma.

Quanto à seleção das empresas, foram predefinidos critérios como: o estágio da implantação do programa; o fato de pertencerem a segmentos de mercado distintos; a disposição para colaborar com a pesquisa; a facilidade de acesso às fontes de evidências; e a similaridade potencial compatível com os casos descritos na literatura. As quatro subsidiárias brasileiras de multinacionais americanas estudadas que satisfizeram os referidos critérios são empresas de grande porte que ocupam posição de destaque no mercado nacional.

Para facilitar o registro das informações e aumentar a confiabilidade dos resultados, as entrevistas foram gravadas, posteriormente transcritas, organizadas e preparadas em uma base de dados textual para análise cruzada dos resultados. Dando um enfoque maior a cada tópico de interesse na análise, foi possível avaliar a influência e implicações de cada um dos fatores do modelo de referência sobre os resultados do Seis Sigma nessas empresas.

A seção seguinte apresenta os resultados e a análise efetuada para o estudo com as quatro empresas, as quais serão denominadas aqui por empresas E1, E2, E3 e E4, respectivamente.

\section{Resultados e análise}

\subsection{Caracterização geral das empresas}

A empresa E1 pertence ao setor metal mecânico e realiza operações na manufatura de elementos de fixação para a indústria de autopeças. Seus principais clientes são montadoras de automóveis, como a Ford, Fiat, GM, Volkswagen, Honda, TRW, entre outras. A posição de líder no mercado nacional, com uma fatia de aproximadamente $25 \%$, permite um faturamento anual em torno de US\$ 35 milhões, como resultado da produção de duas fábricas que trabalham com um contingente de 550 funcionários.

A empresa E2 opera no setor de serviços e efetua transações financeiras, comercializa cartões de crédito e fornece serviços de turismo e viagens de negócios. Os clientes da empresa E2 são portadores de cartão de crédito, tanto pessoas físicas como pessoas jurídicas e estabelecimentos que aceitam seu cartão. É uma das poucas empresas do setor que faz a gestão dos dois grupos de clientes (portadores e estabelecimentos) e tem o cartão de crédito corporativo como um de seus produtos mais fortes. A unidade estudada possui 1200 funcionários que atuam mais diretamente nas operações relacionadas aos cartões de compra e crédito, cheques de viagem e serviços corporativos.

A empresa E3 atua com grande potencial no setor metal mecânico (indústria automotiva), na manufatura de componentes mecânicos e elétricos para veículos de transporte de passageiros e cargas (aviões, automóveis e caminhões). Seus principais clientes são as montadoras de automóveis (Ford, GM, Nissan e VW), os fabricantes de caminhões leves, médios e pesados (GM, Daymler Chrysler e Volvo), além dos fabricantes de maquinário e implementos agrícolas pesados. Em termos de mercado, a empresa E3 detém uma fatia de $24 \%$ no fornecimento de transmissões para veículos de passageiros, já em transmissões leves a participação atinge $47 \%$, sendo que já conseguiu atingir $86 \%$ de participação em transmissões médias. Com relação ao segmento de transmissões pesadas sua participação é de $26 \%$, no entanto, no mercado agrícola chega a não ter concorrentes de destaque. Aproximadamente 2800 funcionários compõem a mão-de-obra direta da empresa.

A empresa E4 faz parte de um grupo multi industrial líder em ciência e tecnologia no desenvolvimento e produção de um portfólio de produtos químicos, plásticos e agrícolas. Com aproximadamente 300 funcionários distribuídos em cinco fábricas em um mesmo site, a empresa $\mathrm{E} 4$ busca equilibrar responsabilidade econômica, ambiental e social. Sua missão é "melhorar constantemente o que é essencial para o progresso humano dominando ciência e tecnologia".

\subsection{Os investimentos no Seis Sigma}

Em termos de investimentos no Seis Sigma, as quatro empresas descritas acima possuem similaridades e peculiaridades quanto aos objetivos, estrutura e estratégia de desdobramento do Seis Sigma. A Tabela 2 expõe resultados obtidos acerca das características gerais de cada uma das empresas estudadas.

Os resultados expostos de forma comparativa na Tabela 2 sugerem que a forma de implementação não está associada ao setor econômico ou ao propósito da implementação do programa Seis Sigma. Nos casos 
Tabela 2. Características gerais do Seis Sigma nas empresas estudadas.

\begin{tabular}{|c|c|c|c|c|}
\hline \multirow[t]{2}{*}{ Características } & \multicolumn{4}{|c|}{ Empresa } \\
\hline & E1 & $\mathbf{E 2}$ & E3 & E4 \\
\hline Início do Seis Sigma & 2002 & 2000 & 1999 & 1999 \\
\hline $\begin{array}{l}\text { Propósito do } \\
\text { investimento no Seis } \\
\text { Sigma }\end{array}$ & $\begin{array}{l}\text { Enfocar mercado e clien- } \\
\text { te; melhorar a qualidade; } \\
\text { inovar; } \\
\text { consolidar a marca; e } \\
\text { voltar-se para a excelên- } \\
\text { cia em gestão. }\end{array}$ & $\begin{array}{l}\text { Meio para obtenção de } \\
\text { resultados sustentáveis } \\
\text { pela maior satisfação dos } \\
\text { clientes. }\end{array}$ & $\begin{array}{l}\text { Busca por melhoria da } \\
\text { qualidade e redução de } \\
\text { custos de produção. }\end{array}$ & $\begin{array}{l}\text { Aumentar desempenho, } \\
\text { produtividade e valor, } \\
\text { além de desenvolver } \\
\text { soluções inovadoras. }\end{array}$ \\
\hline $\begin{array}{l}\text { Forma de implementação } \\
\text { do programa }\end{array}$ & $\begin{array}{l}\text { Programa corporativo } \\
\text { com propriedades de } \\
\text { programa estratégico e } \\
\text { operacional. }\end{array}$ & $\begin{array}{l}\text { Programa corporativo } \\
\text { com propriedades de } \\
\text { programa estratégico e } \\
\text { operacional. }\end{array}$ & $\begin{array}{l}\text { Programa corporativo } \\
\text { com propriedades de } \\
\text { programa estratégico e } \\
\text { operacional. }\end{array}$ & $\begin{array}{l}\text { Programa corporativo } \\
\text { com propriedades de } \\
\text { programa estratégico e } \\
\text { operacional. }\end{array}$ \\
\hline $\begin{array}{l}\text { Estratégia de desdobra- } \\
\text { mento do Seis Sigma } \\
\text { pela denominação de } \\
\text { Sanders e Hild (2000) }\end{array}$ & $\begin{array}{l}\text { Organização de engenha- } \\
\text { ria Seis Sigma. }\end{array}$ & $\begin{array}{l}\text { Organização de engenha- } \\
\text { ria Seis Sigma. }\end{array}$ & $\begin{array}{l}\text { Organização de engenha- } \\
\text { ria Seis Sigma. }\end{array}$ & Organização Seis Sigma. \\
\hline Treinamento da equipe & Pela própria empresa & Por consultoria externa & Pela própria empresa & Pela própria empresa \\
\hline $\begin{array}{l}\text { Encarregado pela } \\
\text { definição de projetos }\end{array}$ & $\begin{array}{l}\text { gerente geral da fábrica, } \\
\text { apoiado por master black } \\
\text { belt }\end{array}$ & gerente de área & $\begin{array}{l}\text { diretor e gerente de } \\
\text { fábrica }\end{array}$ & $\begin{array}{l}\text { diretor do site, master } \\
\text { black belt e gerente de } \\
\text { área }\end{array}$ \\
\hline Pessoas treinadas & $\begin{array}{l}3 \text { black belts } \\
9 \text { green belts }\end{array}$ & $\begin{array}{l}20 \text { black belts } \\
45 \text { green belts }\end{array}$ & $\begin{array}{l}4 \text { black belts } \\
66 \text { green belts }\end{array}$ & $\begin{array}{l}10 \% \text { do público-alvo de } \\
\text { black belts }-70 \% \text { são } \\
\text { green belts }\end{array}$ \\
\hline
\end{tabular}

estudados, as empresas adotaram a mesma forma de implementação (programa corporativo com propriedades de programa estratégico e operacional), mas buscaram propósitos distintos que refletem preocupações estratégicas específicas de cada uma delas em relação às suas posições de mercado e ao modo que visualizaram o impacto no cliente. Além disso, as empresas têm um propósito comum de aumentar o desempenho organizacional, amarrado à necessidade de obtenção de resultados sustentáveis e ao retorno dos investimentos feitos para impulsionar a implantação do Seis Sigma.

Quanto à estratégia de desdobramento do programa, percebe-se um predomínio do tipo "organização de engenharia Seis Sigma", conforme definido por Sanders e Hild (2000). Apenas em E4 a proposta de se tornar uma "organização Seis Sigma" vem sendo almejada, uma vez que se procura fazer com que os conceitos do Seis Sigma sejam disseminados e aplicados na rotina de atividades e não exclusivamente na implementação dos projetos Seis Sigma. Neste sentido, os dados sugerem que a opção pela estratégia do tipo "organização de engenharia Seis Sigma" pode refletir numa disseminação da cultura Seis Sigma mais lenta, com a aplicação do pensamento estatístico mais dificultada. Todavia, tornar-se uma "organização Seis Sigma" parece requerer investimentos mais vultosos no que se refere à formação das pessoas e à criação de uma infra-estrutura tecnológica que facilite a implementação de projetos, como propõe a empresa E4.
Já sobre o estágio de implantação, as empresas E2, E3 e E4 vêm há mais tempo investindo e obtendo significativos resultados. Por esta razão, também possuem mais pessoas treinadas do que E1.

O fato de essas empresas optarem por alocar pessoas da própria empresa para treinar black belts e green belts é um meio de reter ao máximo a visão da empresa sobre o Seis Sigma e de manter mais acentuado o foco nos interesses do negócio, inclusive durante o ensino das metodologias. Para E2, a opção pela consultoria veio em 2005 como medida de economia.

Pelos achados da pesquisa (Tabela 3), as empresas se valem de uma combinação harmônica da abordagem estratégica com a abordagem estatística como elemento primário para estruturar a implantação do programa Seis Sigma, o que reflete a visão conceitual contemporânea do Seis Sigma. Isto fica mais evidente ao se analisar a forma adotada pelas empresas para definirem os projetos Seis Sigma, por sua orientação voltada para o mercado e pelo uso dos resultados dos projetos como direcionadores de desempenho do negócio.

A forma adotada pelas organizações para definir os projetos Seis Sigma é um dos pontos fundamentais da estrutura de implantação do programa de forma geral. Pela Tabela 3, nas quatro empresas, o direcionamento estratégico dos projetos está embasado em metas de desempenho do negócio pré-estabelecidas pela alta liderança corporativa; o que reforça a proposição de que a definição dos projetos Seis Sigma decorre do alinha- 
Tabela 3. Aplicabilidade dos fatores essenciais do Modelo de Referência em E1, E2, E3 e E4.

\begin{tabular}{|c|c|c|c|c|}
\hline \multirow[t]{2}{*}{ Fator essencial } & \multicolumn{4}{|c|}{ Empresa } \\
\hline & E1 & E2 & $\mathbf{E 3}$ & E4 \\
\hline $\begin{array}{l}\text { Direcionamento e foco } \\
\text { dos projetos (orientação e } \\
\text { alinhamento estratégico) }\end{array}$ & $\begin{array}{l}\text { O alinhamento estratégi- } \\
\text { co é priorizado; devem } \\
\text { impactar alavancadores } \\
\text { do negócio; devem atin- } \\
\text { gir metas financeiras e de } \\
\text { redução de defeitos }\end{array}$ & $\begin{array}{l}\text { O alinhamento estratégi- } \\
\text { co é priorizado; devem } \\
\text { impactar o cliente e } \\
\text { melhorar os processos } \\
\text { críticos; focados em qua- } \\
\text { lidade e definem metas } \\
\text { de redução de falhas }\end{array}$ & $\begin{array}{l}\text { Devem impactar cate- } \\
\text { gorias de desempenho } \\
\text { do negócio pré-estabele- } \\
\text { cidas; pouca ênfase em } \\
\text { metas financeiras; visam } \\
\text { reduzir custo operacional }\end{array}$ & $\begin{array}{l}\text { Refletem oportunidades } \\
\text { já identificadas; devem } \\
\text { impactar em medidas de } \\
\text { desempenho do negó- } \\
\text { cio, no cliente e gerar } \\
\text { resultados financeiros } \\
\text { significativos }\end{array}$ \\
\hline $\begin{array}{l}\text { Uso de medidas de } \\
\text { desempenho no controle } \\
\text { gerencial do programa } \\
\text { Seis Sigma }\end{array}$ & $\begin{array}{l}\text { Controle feito por } \\
\text { indicadores financeiros e } \\
\text { não financeiros inseridos } \\
\text { em um SMD estrutura- } \\
\text { do e multidimensional } \\
\text { corporativo }\end{array}$ & $\begin{array}{l}\text { Controle feito por indi- } \\
\text { cadores financeiros e não } \\
\text { financeiros inseridos em } \\
\text { SMD multidimensional } \\
\text { corporativo }\end{array}$ & $\begin{array}{l}\text { Controle feito por } \\
\text { indicadores financeiros e } \\
\text { não financeiros inseridos } \\
\text { em um SMD estrutura- } \\
\text { do e multidimensional } \\
\text { corporativo }\end{array}$ & $\begin{array}{l}\text { Controle feito por indi- } \\
\text { cadores financeiros e não } \\
\text { financeiros inseridos em } \\
\text { um SMD do negócio }\end{array}$ \\
\hline $\begin{array}{l}\text { Uso de estatística (pensa- } \\
\text { mento estatístico) }\end{array}$ & $\begin{array}{l}\text { Facilita a comunicação } \\
\text { e o entendimento dos mé- } \\
\text { todos estatísticos }\end{array}$ & $\begin{array}{l}\text { Facilita a disseminação } \\
\text { da cultura e da metodolo- } \\
\text { gia Seis Sigma }\end{array}$ & $\begin{array}{l}\text { Serve de subsídio básico } \\
\text { para o entendimento da } \\
\text { metodologia Seis Sigma }\end{array}$ & $\begin{array}{l}\text { Conceitos valorizados } \\
\text { que contribuem para o } \\
\text { uso da estatística na aná- } \\
\text { lise de dados em geral }\end{array}$ \\
\hline $\begin{array}{l}\text { Metodologias utilizadas } \\
\text { na implementação dos } \\
\text { projetos }\end{array}$ & $\begin{array}{l}\text { DMAIC; DFSS; } \\
\text { Ferramentas de lean } \\
\text { manufacturing }\end{array}$ & DMAIC; DFSS & $\begin{array}{l}\text { DMAIC; } \\
\text { Ferramentas de lean } \\
\text { manufacturing }\end{array}$ & $\begin{array}{l}\text { DMAIC; DFSS; } \\
\text { 3I-Implement/ improve/ } \\
\text { inovate. }\end{array}$ \\
\hline $\begin{array}{l}\text { Capacitação/ } \\
\text { especialização do black } \\
\text { belt }\end{array}$ & $\begin{array}{l}\text { Mostra capacidade de } \\
\text { liderança e visão do } \\
\text { negócio e da corporação; } \\
\text { lidera times; treina e } \\
\text { orienta green belts; deve } \\
\text { atingir metas financeiras } \\
\text { com projetos; e atua em } \\
\text { tempo integral. }\end{array}$ & $\begin{array}{l}\text { Mostra capacidade de } \\
\text { liderança e de gerenciar } \\
\text { processos; tem visão } \\
\text { sobre o negócio; lidera } \\
\text { times; treina e orienta } \\
\text { green belts; dá suporte } \\
\text { técnico em análise de } \\
\text { dados; e atua em tempo } \\
\text { integral. }\end{array}$ & $\begin{array}{l}\text { Mostra capacidade de } \\
\text { liderança e de gestão de } \\
\text { processo; tem visão geral } \\
\text { do negócio; auxilia na } \\
\text { formação de green belts } \\
\text { e yellow belts; e atua em } \\
\text { tempo integral. }\end{array}$ & $\begin{array}{l}\text { Mostra capacidade de } \\
\text { liderança, de comuni- } \\
\text { cação e de gerência de } \\
\text { processos; tem visão } \\
\text { do negócio; auxilia na } \\
\text { formação de green belts; } \\
\text { e atua em tempo parcial. }\end{array}$ \\
\hline $\begin{array}{l}\text { Suporte de TI para gerên- } \\
\text { cia dos projetos }\end{array}$ & $\begin{array}{l}\text { Possui um sistema cor- } \\
\text { porativo na intranet para } \\
\text { gerenciar os projetos e os } \\
\text { resultados do programa } \\
\text { Seis Sigma } \\
\end{array}$ & $\begin{array}{l}\text { Possui um sistema cor- } \\
\text { porativo na intranet para } \\
\text { gerenciar os projetos e os } \\
\text { resultados do programa } \\
\text { Seis Sigma } \\
\end{array}$ & $\begin{array}{l}\text { Possui um sistema de } \\
\text { informação para controle } \\
\text { dos resultados do Seis } \\
\text { Sigma inserido no SMD } \\
\text { do negócio }\end{array}$ & $\begin{array}{l}\text { Possui um sistema de in- } \\
\text { formação para gerenciar } \\
\text { o portfólio de projetos e } \\
\text { de resultados do progra- } \\
\text { ma Seis Sigma }\end{array}$ \\
\hline $\begin{array}{l}\text { Fatores críticos de } \\
\text { sucesso }\end{array}$ & $\begin{array}{l}\text { Sustentar os investimen- } \\
\text { tos; comprometimento do } \\
\text { time; eficiência geren- } \\
\text { cial; e divulgação de } \\
\text { resultados }\end{array}$ & Apoio gerencial & $\begin{array}{l}\text { Disseminação da cultura; } \\
\text { sustentar os investimen- } \\
\text { tos para formação das } \\
\text { pessoas }\end{array}$ & $\begin{array}{l}\text { Alinhamento estratégi- } \\
\text { co; suporte gerencial; } \\
\text { produzir economias } \\
\text { significativas }\end{array}$ \\
\hline
\end{tabular}

mento estratégico como ponto de partida (orientação top-down). Assim, os projetos têm impacto direto nos elementos críticos do negócio, geram melhoria significativa dos processos afetados por sua implementação, proporcionam economias e redução de custos, aumentam a satisfação do cliente, entre outros. No caso de E4, percebeu-se mais flexibilidade em termos de origem das propostas de projetos. Como a empresa contempla em sua estrutura um portfólio de projetos sugeridos pelos especialistas, que reflete a identificação de oportunidades no ambiente operacional, há mais flexibilidade e a orientação botton-up para definição também é utilizada. No entanto, o alinhamento é um aspecto prioritário para selecionar um projeto sugerido dessa forma.
Os resultados fornecem subsídios de que a priorização do alinhamento estratégico independe da estratégia de desdobramento adotada, e que o direcionamento dos projetos é determinado por pessoas de posição de alta liderança e pelos master black belts, a fim de que o foco estratégico dos projetos seja garantido. Ademais, quando há alinhamento estratégico na definição dos projetos e a implementação é efetivada, o feedback estratégico acontece e abre a possibilidade de direcionamento de outros projetos. Vale ressaltar que, apesar da ênfase em resultados financeiros ressaltada nas publicações do período de 1992 a 2000, não houve evidências empíricas de que este seja um pressuposto válido para definir projetos Seis Sigma. 
Sobre o uso de estatística, os resultados dão indicativos de que a aplicação do pensamento estatístico contribui para facilitar a disseminação da cultura e dos conceitos estatísticos essenciais, para que a equipe utilize uma linguagem comum de comunicação, aumentando ainda mais o potencial da metodologia Seis Sigma e fortalecendo o embasamento necessário para a definição e implementação dos projetos. É o alicerce necessário para formação dos especialistas.

No que tange aos aspectos metodológicos, os resultados indicam que os especialistas se restringem às aplicações dos métodos e técnicas assimilados durante o treinamento, os quais estão mais focados no DMAIC e, em algumas situações, no DFSS.

A experiência da empresa E3 tem mostrado que a implementação de projetos Seis Sigma não depende da aplicação de métodos estatísticos como um requisito da implementação dos projetos. A aplicabilidade das ferramentas demanda, sobretudo, o conhecimento mais detalhado das funções das ferramentas e do alcance que elas podem ter em termos de redução de defeitos, de eficiência, de eficácia dos processos e de economias proporcionadas.

Embora o uso das ferramentas estatísticas básicas inseridas no ciclo DMAIC seja enfatizado na empresa E4, em algumas áreas funcionais como, por exemplo, segurança do trabalho, há maior dificuldade de aplicação dos métodos pela especificidade das variáveis. A empresa E1 é a que usa mais intensamente o DFSS e o emprega em projetos para desenvolvimento de novas ferramentas para produção dos elementos de fixação.

Nas empresas E1 e E3, as duas fornecedoras da indústria automotiva, têm sido mais intenso o uso dos princípios e ferramentas de lean manufacturing (GODINHO FILHO; FERNANDES, 2004) com o intuito de melhorar o potencial produtivo, principalmente focando a redução de custos. A eliminação de desperdícios é o princípio atacado mais de perto pelas empresas, buscando manter um alinhamento com as metas do Seis Sigma. A redução de custos e a geração de recursos financeiros e intelectuais são aspectos priorizados neste contexto, tendo em vista que eliminar fontes de variabilidade que causem efeitos indesejáveis sobre os custos de produção, instabilidade, retrabalho, insatisfação dos clientes, entre outros pontos, provocam perda de oportunidades de melhoria do desempenho organizacional. A incorporação desta visão incrementa o valor dos resultados decorrentes da metodologia Seis Sigma. Numa linha alternativa, a empresa E4 tem investido no desenvolvimento de uma metodologia própria (3I) para gerar soluções inovadoras.

A aplicação da metodologia sistematizada, como já era esperado, revelou ser um dos pontos fortes do sucesso do Seis Sigma, juntamente com o aumento do capital humano decorrente da melhor capacitação das pessoas na utilização dos métodos científicos.

Sobre o suporte de TI, os resultados reforçam a literatura. É notório que não basta uma estratégia de desdobramento condizente com os recursos materiais, humanos e financeiros. O gerenciamento efetivo do programa Seis Sigma depende de alto investimento em tecnologia da informação, aliado ao uso de soluções computacionais apropriadas. Considerando esse fator crítico de sucesso, todas as empresas estudadas investiram no desenvolvimento de sistemas web destinados a capturar e impulsionar a aquisição de conhecimento. Os sistemas dispõem de vários indicadores que auxiliam no gerenciamento dos projetos e na identificação de oportunidades para novos projetos.

Nesta linha, os achados deste estudo vão ao encontro do que apontaram Ingle e Roe (2001) e Laurindo (2002), quando ressaltaram que a infra-estrutura de TI combinada ao uso apropriado das ferramentas estatísticas vêm se estabelecendo como um novo fator crítico de sucesso do Seis Sigma.

O uso dos indicadores de desempenho, apoiado no suporte de TI como um recurso tecnológico importante para um melhor controle dos resultados do programa Seis Sigma, também é valorizado nas empresas E1, E2, E3 e E4. Cada empresa possui um sistema de informação corporativo que permite o acompanhamento das atividades relativas à implementação do programa, e estas possibilitam o feedback estratégico representado pelos indicadores de desempenho do negócio inseridos no sistema de medição de desempenho multidimensional. No caso de E1, o SMD está estruturado por um balanced scorecard com quatro perspectivas. Já em E3, o sistema foi concebido a partir dos requisitos do Prêmio Malcolm Baldrige dos Estados Unidos. E4 possui seu portfólio de projetos inserido no sistema de controle corporativo, o qual pode ser alimentado com sugestões de projetos encaminhados por black belts ou green belts. Tais sugestões são analisadas por master black belts e por pessoas da alta liderança quanto à sua viabilidade e alinhamento com os interesses do negócio.

Foi importante verificar que em todas as empresas o sucesso da implementação dos projetos Seis Sigma tende a ser usado como veículo importante para melhorar a mensuração dos indicadores de desempenho, de modo a suprir o SMD com informações relevantes para uma possível revisão e/ou redirecionamento de metas e objetivos de médio e longo prazo.

Os elementos apontados pelos black belts e green belts como fatores críticos de sucesso reforçam a posição de Motwani et al. (2004), quando destaca que o sucesso da implantação do programa Seis Sigma requer investimentos em planejamento, esforço e flexibilidade. Embora outros fatores tenham sido apontados como determi- 
nantes no sucesso do programa, como: sustentação dos investimentos, produção e divulgação dos resultados, comprometimento do time, mudança de cultura, entre outros que dependem das peculiaridades do negócio de cada empresa, o suporte gerencial parece ser o mais preponderante sob a óptica dos especialistas.

De forma geral, os resultados obtidos nesta pesquisa fornecem indicativos de que os fatores que compõem o modelo de referência possuem uma contribuição efetiva para o sucesso e consolidação do Seis Sigma nas empresas estudadas. Este resultado aponta para um aspecto importante acerca da aplicação da metodologia Seis Sigma como fator primordial para caracterizar o sucesso do programa Seis Sigma. Os resultados obtidos ressaltam sua necessidade, mas não sua suficiência, e apontam para o caráter essencial de outros fatores salientados no modelo de referência, que não devem ser negligenciados em qualquer etapa do investimento no Seis Sigma.

Além disso, os estudos de caso esclareceram melhor como a combinação das abordagens estatística e estratégica potencializa o papel integrador de objetivos estratégicos e operacionais desempenhado pelo Seis Sigma.

\section{Considerações finais}

Este trabalho traz parte dos resultados de uma pesquisa mais abrangente que avaliou várias implicações teóricas e evidências empíricas acerca de fatores críticos para o sucesso da implementação do programa Seis Sigma.

O modelo de referência, por ser um modelo fundamentado na literatura e fortalecido por evidências empíricas advindas de empresas onde o Seis Sigma está consolidado, encaminha o aprimoramento da implementação do programa Seis Sigma à medida que fornece a possibilidade das organizações avaliarem o sucesso do Seis Sigma pela óptica dos fatores críticos. É importante salientar que esta proposta não contempla a criação de uma normatização sobre a forma de implementação do programa Seis Sigma. De fato, o modelo de referência constitui uma alternativa para orientar uma empresa a estruturar ou reestruturar a implementação do programa Seis Sigma, estimulando uma reflexão sobre opções e perspectivas estratégicas que podem conduzir ao aumento do desempenho organizacional.

Os resultados obtidos sugerem que os princípios de lean manufacuring, como parte do contexto metodológico do Seis Sigma, podem ser incluídos de forma mais explícita no modelo de referência. Isto é um indicativo de que a incorporação de outras teorias ao contexto do Seis Sigma é uma tendência atual que precisa ser mais explorada a fim de aumentar seu potencial estratégico.

Muito embora os resultados dos estudos de caso tenham contribuído para esclarecer aspectos pouco explorados na literatura no contexto das evidências empíricas sobre o Seis Sigma, retratando uma parte do cenário de empresas que atuam no Brasil, e também reforçando a essência do modelo de referência, ainda não foram elucidadas dúvidas acerca do efeito dos investimentos no Seis Sigma sobre o desempenho organizacional no longo prazo. Apesar dos benefícios já identificados para as organizações precursoras no assunto, ainda falta um melhor posicionamento acerca da eficiência do Seis Sigma para as empresas do Brasil. Na agenda de trabalhos futuros pretende-se obter resultados mais concretos e conclusivos sobre esta questão, por meio da análise de indicadores de desempenho. 


\title{
Reference model to structure the Six Sigma in organizations
}

\begin{abstract}
This paper introduces the reference model to structure Six Sigma. This model is a result of theory incorporation that contributes to increase the strategic power of Six Sigma for improving businesses performance. Reference model proposal points out certain primordial requirements for de Six Sigma program success. The theoretical basis to sustain the reference model was supported in studies about the influence of critical factors such as: strategic orientation and strategic alignment; business performance measurement; statistical approach (statistical thinking); people training; project implementation; and information technology use. Complementing the model proposition, this paper presents an empiric point of view about the contribution factors examined in the reference model formulation. The results show case studies carried out in four Brazilian subsidiaries of large multinationals companies. The case studies analysis indicates some positive evidences of the considerable influence of the previously mentioned factors on Six Sigma success and consolidation.
\end{abstract}

Keywords: Six Sigma. Quality. Strategic alignment. Performance measurement. DMAIC. Statistical thinking.

\section{Referências bibliográficas}

ANTONY, J. Some pros and cons of six sigma: an academic perspective. The TQM Magazine, York, England, v.16, n.4, p.303-306, 2004.

ARNHEITER, E.; MALEYEFF, J. The integration of lean management and six sigma. The TQM Magazine, York, England, v.17, n.1, p.5-18, 2005.

BEHARA, R. S.; FONTENOT, G. F.; GRESHAM, A. Customer satisfaction measurement and analysis using six sigma. International Journal of Quality \& Reliability Management, Bradford, England, v.12, n.3, p.9-18, 1995.

BLAKESLEE Jr, J.A. Implementing the six sigma solution. Quality Progress, New York, v.32, n.7, p.77-85, July 1999.

BRYMAN, A. Research methods and organization studies. London: Unwin Hyman, 1989. 283p.

CORONADO, R. B.; ANTONY, J. Critical success factors for the successful implementation of six sigma projects in organisations. The TQM Magazine, York, England, v.14, n.2, p.92-99, 2002.

CRESWELL, J. W. Research design: qualitative \& quantitative approaches. London: SAGE Publications, 1994, 228p.

CROSS, K. F.; LYNCH, R. L. Managing the corporate warriors. Quality Progress, New York, v.23, n.4, p.54-59, 1990.

DALE, B. G.; WILLIAMS, R. T.; WIELE, T. Marginalization of quality: is there a case to answer? The TQM Magazine, York, England, v.12, n.4, p.266-274, 2000.

DANSKY, K. H.; BRANNON, D. Strategic orientation and TQM: linking vision to action. Journal of Quality Management, Greenwich, v.1, n.2, p.227-242, 1996.

DRANSFIELD, S. B.; FISHER, N. I.; VOGEL, N. J. Using statistics and statistical thinking to improve organizational performance. International Statistical Review, Edinburgh, England, v.67, n.2, p.99-150, 1999.

ECKES, G. A revolução seis sigma. Rio de Janeiro: Campus, 2001, 272p.

EDVINSSON, L.; MALONE, M. S. Capital intelectual. São Paulo: Makron Books, 1998. 214p.

EVANS, J. R. An exploratory study of performance measurement systems and relationships with performance results. Journal of
Operations Management, Amsterdam, v.22, n.3, p.219-223, 2004.

GEORGE, M. L. Lean seis sigma para serviços. Rio de Janeiro: Qualitymark, 2004. 436p.

GODINHO FILHO, M.; FERNANDES, F. C.F. Manufatura enxuta: uma revisão que classifica e analisa os trabalhos apontando perspectivas de pesquisas futuras. Gestão e Produção, São Carlos, v.11, n.1, p.1-19, 2004.

$\mathrm{GOH}, \mathrm{T}$. N. The role of statistical design of experiments in six sigma: perspectives of a practitioner. Quality Engineering, New York, v.14, n.4, p.659-671, 2002.

GOH, T. N.; XIE, M. Improving on the six sigma paradigm. The TQM Magazine, York, England, v.16, n.4, p.235-240, 2004.

HAHN, G.; DOGANAKSOY, N.; HOERL, R. W. The evolution of six sigma. Quality Engineering, New York, v.12, n.3, p.317-326, 2000.

HARRY, M. J. Six sigma: a breakthrough strategy for profitability. Quality Progress, New York, v.31, n.5, p.60-64, May 1998.

HARRY, M. J.; SCHROEDER, R. Six sigma: the breakthrough management strategy revolutionizing the world's top corporations. New York: Doubleday, 2000, 300p.

HOERL, R. W. Six sigma and the future of the quality profession. Quality Progress, New York, v.31, n.6, p.35-42, 1998.

HOERL, R. W. Six sigma black belts: what do they need to know? Journal of Quality Technology, Milwaukee, v.33, n.4, p.391-406, 2001.

INGLE, S.; ROE, W. Six sigma black belt implementation. The TQM Magazine, York, England, v.13, n.4, p.273-280, 2001.

KWAK, Y. H.; ANBARI, F. T. Benefits, obstacles, and future of six sigma approach. Technovation, Essex, England, v.26, n.5-6, p.708-715, 2006.

LAURINDO, F. J. B. A tecnologia da informação e o Seis Sigma. In: ROTONDARO, R. G. Seis Sigma: estratégia gerencial para melhoria de processos, produtos e serviços. São Paulo: Atlas, p. 358-37, 2002.

McADAM, R.; BAILIE, B. Business performance measures and alignment impact on strategy: the role of business improvement 
models. International Journal of Operations \& Production Management, Wagon Lane, UK, v.22 n.9, p.972-996, 2002.

MITCHELL, B. The Six Sigma appeal. Engineering Management Journal, Stevenage, UK, v.2, n.1, p.41-47, Feb 1992.

MOTWANI, J.; KUMAR, A.J. A business process change framework for examining the implementation of six sigma: a case study of Dow Chemicals. The TQM Magazine, York, England, v.16, n.4, p.273-283, 2004.

NEELY, A. D. Measuring business performance. London: Economist Books, 1998. 208p.

NEELY, A. D. The performance measurement revolution: why now and what next? International Journal of Operations \& Production Management, Bradford, v.19, n.2, p.205-228, 1999.

PANDE, P.; NEUMAN, R. P.; CAVANAGH, R. R. Estratégia Seis Sigma. Rio de Janeiro: Qualitymark Ed., 2001. 442p.

Perez-Wilson, M. Seis Sigma: compreendendo o conceito, as implicações e os desafios. Rio de Janeiro: Qualitymark Ed., 1999. 283p.

REVERE, L.; BLACK, K. Integrating six sigma with total quality management: a case example for measuring medication errors. Journal of Healthcare Management, Chicago, v.48, n.6, p.377-391, 2003.

SANDERS, D.; HILD, C. R. A discussion of strategies for six sigma implementation. Quality Engineering, New York, v.12, n.3, p.303-309, 2000.

SANTOS, A. B. Modelo de Referência para estruturar o programa de qualidade seis sigma: proposta e avaliação. São Carlos, 2006. v.1. Tese - (Doutorado em Engenharia de Produção). Universidade Federal de São Carlos.
SANTOS, A. B.; MARTINS, M. F. A implementação dos projetos seis sigma contribuindo para o direcionamento estratégico e para o aprimoramento do sistema de medição de desempenho. Revista Pesquisa e Desenvolvimento Engenharia de Produção, Itajubá, v.1, n.1, p.1-14, Dez. 2003.

SANTOS, A. B.; MARTINS, M. F. Pensamento estatístico: um componente primordial para o sucesso do programa de qualidade seis sigma. In. ENCONTRO NACIONAL DE ENGENHARIA DE PRODUÇÃO, 24. 2004. Anais... Florianópolis, SC, Nov. 2004. (CD-ROM).

SANTOS, A. B.; MARTINS, M. F. Medição de desempenho e alinhamento estratégico: requisitos para o sucesso do Seis Sigma. In. Simpósio de Administração da Produção, Logística e Operações Internacionais, 7. 2005. Anais... São Paulo, SP, Ago. 2005. (CD-ROM).

SCHROEDER, R. G.; LINDERMAN, K.; LIEDTKE, C.; CHOO, A. S. Six sigma: old wine in new bottles? Working Paper, Carlson School of Management, University of Minnesota, Dec., 2002.

SNEE, R. D. Impact of six sigma on quality engineering. Quality Engineering, New York, v.12, n.3, p. 9-4, 2000.

SULLIVAN, P. H. Profiting form intellectual capital. Journal of Knowledge Management, West Yorkshire, v.3, n.2, p.132-142, 1999.

TADIKAMALLA, P. R. The confusion over six-sigma quality. Quality Progress, New York, v.24, n.11, p.83-85, Nov. 1994.

VALLE, B. M. Tecnologia da informação no contexto organizacional. Ciência da Informação, Brasília, v. 25, n.1, p. 7-11, 1996.

YIN, R. K. Estudo de caso: planejamento e métodos. 3 ed. Porto Alegre: Bookman, 2005. 212p.

\section{Sobre os autores}

\section{Adriana Barbosa Santos}

Departamento de Ciências de Computação e Estatística, Universidade Estadual Paulista - UNESP,

Rua Cristóvão Colombo, 2265, CEP 15054000, São José do Rio Preto, SP, Brasil,

e-mail: adriana@ibilce.unesp.br

\section{Manoel Fernando Martins}

Departamento de Engenharia de Produção, Universidade Federal de São Carlos - UFSCar, Rodovia Washington Luís (SP-310), KM 235, CEP 13565-905, São Carlos, SP, Brasil,

e-mail: manoel@ power.ufscar.br 